\title{
The Four Quadrant-ZOG and Annular Groove Phase Mask Coronagraphs
}

\author{
Dimitri Mawet $^{a}$, Pierre Riaud ${ }^{a}$, Olivier Absil ${ }^{a}$, Jacques Baudrand $^{b}$ and Jean Surdej ${ }^{a}$ \\ ${ }^{a}$ University of Liège, 17 Allée du 6 Août, B-4000 Sart Tilman, Belgium; \\ ${ }^{b}$ Observatory of Paris-Meudon, 5 Place Jules Janssen, F-92195 Meudon, France
}

\begin{abstract}
Phase mask coronagraphy is a technique aiming at accommodating both high dynamic and high angular resolution imaging of faint sources around bright objects such as exo-planets orbiting their parent stars or host galaxies around Active Galactic Nuclei. We present two new phase mask coronagraphs implemented with subwavelength diffractive optical elements consisting of optimized surface-relief gratings. The first one is an evolution of the Four Quadrant Phase Mask coronagraph, which resolves the $\pi$ phase shift chromaticity problem: the Four Quadrant Zeroth Order Grating (4QZOG). The second one is a totally new design consisting of an optical vortex induced by a space-variant grating: the Annular Groove Phase Mask (AGPM) coronagraph is fully symmetric and free from any "shaded zones". Some manufacturing hints are given.
\end{abstract}

Keywords: Phase mask coronagraphy, achromatic phase shifters, subwavelength gratings, space-variant gratings, optical vortices

\section{INTRODUCTION}

Direct detection of faint sources around bright astrophysical objects like stars or Active Galactic Nuclei is very difficult due to the large flux ratio between them. For example, an Earth-like exoplanet is typically $10^{9}$ times fainter than its host star in the visible, $10^{6}$ in the thermal infrared while the contrast of already known debris disks around main sequence stars is generally larger than one thousand in the visible (e.g. the $\beta$-Pictoris's disk, see Smith \& Terrile $^{1}$ ). The circumnuclei structures of AGN (obscuring torus, jet-induced structures,...) are at least one hundred times less luminous than the central engine at visible and near-IR wavelengths (e.g. NGC 1068, see Gratadour et al. $^{2}$ for instance). The study of such objects therefore requires dedicated instruments such as coronagraphs. Coronagraphy has been used in high dynamical imaging since its invention by Bernard Lyot in 1939 (see Ref. 3). The Lyot coronagraph principle is very simple: it consists of an occulting mask at the telescope focus which is centered on the bright object and a well-dimensioned diaphragm, the so-called Lyot Stop, in the relayed pupil to remove diffraction residuals. Lyot's design proved to be efficient for solar and stellar coronagraphy and prolific, giving birth to an entire new family of improved by-products, the so-called amplitude coronagraphs. Unfortunately, amplitude coronagraphs possess a major inherent weakness: the physical extension of the opaque zone occults quite a significant region centered on the optical axis and thus all the sources behind it. For example, let us mention the case of the recent Notch-Filter Mask ${ }^{4}$ : this design presents a large central opaque zone extending in the focal plane up to $3 \lambda / D$ ( $D$ is the telescope diameter whereas $\lambda$ is the wavelength) where the potential companion is still attenuated by at least $50 \%$. New types of coronagraphs have been designed as alternative solutions to the classical amplitude coronagraphs. This new family of components acts on the phase instead of acting on the amplitude, they are therefore called phase coronagraphs. ${ }^{5}$ The phase coronagraphs are not affected by the inherent central "dead zone" of the amplitude coronagraphs. Rouan et al. ${ }^{6}$ proposed in 2000 a very performing design, the so-called four-quadrant phase mask coronagraph (FQ-PM). The principle is to divide the focal plane in four equal areas centered on the optical axis, with two of them providing a $\pi$ phase shift. This causes destructive interference ("nulling") to occur inside the geometric pupil area (Fig. 1). The nulling phenomenon's quality determines the coronagraph performance and is quantified by the value of the so-called "nulling (or null) depth" coefficient over the considered spectral band. The FQ-PM principle has

Further author information: (Send correspondence to Dimitri Mawet)

Dimitri Mawet: E-mail: mawet@astro.ulg.ac.be, Telephone: +32 (4) 3669761 


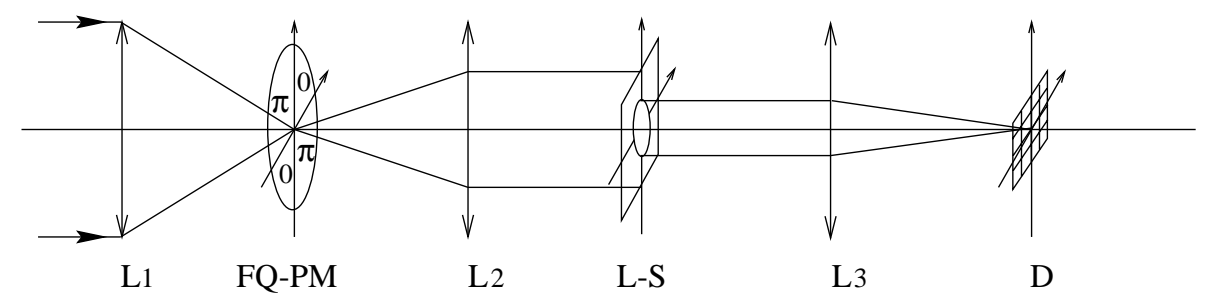

Figure 1. Common coronagraphic optical bench scheme shown here in the FQ-PM case: L1, L2 and L3 are three lenses in the optical system. L1 provides a large (to minimize spatial defects) $F / D$ ratio on the coronagraph; L2 images the pupil in the second plane. The Lyot Stop (L-S) suppresses the diffracted starlight, and finally L3 forms the coronagraphic image on the detector D.

been validated on the bench in monochromatic light ${ }^{9}$ and installed on the NAOS-CONICA adaptive optics at the VLT (Very Large Telescope) UT4. It has given promising preliminary scientific results ${ }^{2}$ and perspectives for future instruments such as JWST ${ }^{10}$ (the infrared successor of the Hubble Space Telescope) and VLT-PF ${ }^{11}$ (Planet-Finder, a proposal for the second generation instruments of the Very Large Telescope). In the phase coronagraph family, let us also mention the special cases of the $\mathrm{AIC}^{7}$ (Achromatic Interferometric Coronagraph) which consists in a single pupil achromatic nulling interferometer and the so-called "vortex spatial filter", 8 which is a monochromatic pupil plane mask.

Manufacturing challenges of the FQ-PM come from the $\pi$ phase shift achievement. So far, only monochromatic masks have been realized using thin film deposition or etching techniques on the "index step" principle: a step height $h$ in a material of refractive index $n$ at the wavelength $\lambda$ induces a phase shift:

$$
\Delta \phi=\frac{2 \pi}{\lambda}(n-1) h
$$

This only works perfectly for a given wavelength. The hyperbolic phase shift dependence in $\lambda$ makes unapplicable the high performance use of such devices over a large spectral bandwidth. For instance, the nominal performance of the monochromatic mask used on NAOS-CONICA in the K band $(2-2.4 \mu \mathrm{m})$ under well-corrected atmospheric turbulence is about two thousand times weaker than in monochromatic laboratory experiments. In this paper, we present an original solution to achromatize and implement the $\pi$ phase shift on the FQ-PM so it could be used over regular spectral bands corresponding to classical astrophysical filters allowing high nulling performances. Large band observations permit shorter integration times and object characterizations by means of spectroscopic analysis. We propose a solution based on the dispersion of the so-called form birefringence of subwavelength surface-relief gratings. We demonstrate that their fruitful optimized implementation into a four quadrant scheme resolves the chromaticity problem down to an acceptable level. Unfortunately, the FQ-PM will always possess one drawback: the four phase transitions between adjacent quadrants create four $\lambda / D$-large "shaded zones" where the potential circumstellar signal or companion is attenuated by up to 4 magnitudes. ${ }^{12}$ We therefore propose a new design of phase mask coronagraph derived from the FQ-PM that totally suppresses its annoying "shaded zones" and inherently allows to reduce the chromaticity issues in the same way. This new coronagraph is referred to as the Annular Groove Phase Mask coronagraph (AGPM) in reference to the concentric circular subwavelength grating it is made of.

\section{SUBWAVELENGTH GRATINGS / ZOGS}

When the period $\Lambda$ of the grating is smaller than the wavelength of the incident light, it does not diffract as a classical spectroscopic grating. All the incident energy is enforced to propagate only in the zeroth order, leaving incident wavefronts free from any further aberrations. The subwavelength gratings are therefore often called Zeroth Order Gratings (ZOGs). Whether a diffraction order propagates or not is determined by the well-known grating equation from which a "ZOG condition" on the grating period to wavelength ratio can be derived:

$$
\frac{\Lambda}{\lambda} \leq \frac{1}{n_{I} \sin \chi+\max \left(n_{I}, n_{I I I}\right)}
$$


where $\chi$ is the angle of incidence, $n_{I}$ and $n_{I I I}$ are the refractive indices of the incident (superstrate) and transmitting (substrate) media, respectively (see Fig. 2). This type of gratings behaves like homogeneous media with unique characteristics, which can be used to synthesize artificial birefringent achromatic waveplates ${ }^{13,14}$ or monolithic anti-reflective structures. ${ }^{15}$ Quarterwave or halfwave plates are extensively used in astrophysics for polarimetric studies. Subwavelength gratings constitute an elegant and flexible solution to produce them. The

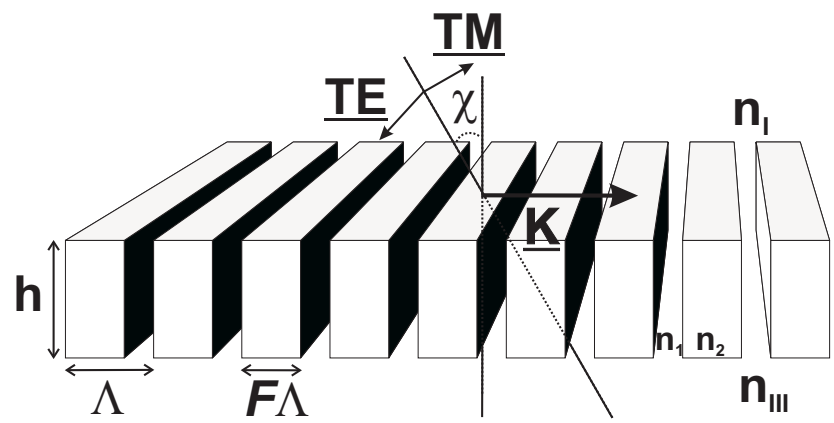

Figure 2. ZOG schematic presenting the main parameters of the grating: the grating vector $|\underline{K}|=2 \pi / \Lambda$, perpendicular to the grating lines, with $\Lambda$ being the period, the grating depth $h$ and the so-called filling factor $F$, such that $F \Lambda$ is the width of the grating ridges. $\underline{T E}$ and $\underline{T M}$ are the vectorial orthogonal polarization components of the $\chi$-incident light. $n_{I}$ and $n_{I I I}$ are the refractive indices of the incident and transmitting media, respectively. $n_{1}$ and $n_{2}$ are the refractive indices of the grating itself (in our case, $n_{1}=n_{I}$ and $n_{2}=n_{I I I}$ ). $\underline{T E}$ is the transverse electric vibration where the electrical field vector is perpendicular to the plane of incidence (the plane of incidence is defined by the grating normal and the direction of the incoming light; in our case by the grating normal and the grating vector) and $\underline{T M}$ is the transverse magnetic one (the electrical field vector lies in the plane of incidence).

key point is that by carefully controlling the geometry of the grating structure (via the grating parameters: the period $\Lambda$, the depth $h$ and the width of the grating ridges $F \Lambda$ with $F$ the filling factor) we are able to tune the so-called form birefringence

$$
\Delta n_{T E-T M}(\lambda)=n_{T E}(\lambda)-n_{T M}(\lambda)
$$

where $n_{T E}$ and $n_{T M}$ are the two effective indices associated with the subwavelength structure, one for each polarization state: $T E$ (tranverse electric, see Fig. 2) and $T M$ (tranverse magnetic). Intuitively, one can understand this artificial anisotropy and the existence of two distinct effective indices: the incident light sees two different media as its vectorial components vibrate parallel or orthogonal to the grating lines. The idea is to make the form birefringence proportional to the wavelength in order to achromatize the subsequent differential $\pi$ phase shift between the two polarization states $T E$ and $T M$

$$
\Delta \phi_{T E-T M}(\lambda)=\frac{2 \pi}{\lambda} h \Delta n_{T E-T M}(\lambda) \approx \pi
$$

with $h$, the optical path through the birefringent medium. This is a good example of refractive index engineering. To simulate grating responses and calculate the form birefringence $\Delta n_{f o r m}=\Delta n_{T E-T M}$ in the subwavelength and resonant domain $(\Lambda \approx \lambda)$, scalar theories of diffraction dramatically fail. The vectorial nature of light must be taken into account implying a resolution of the Maxwell equations by the so-called Rigorous Coupled Wave Analysis $\left(\mathrm{RCWA}^{16}\right)$. RCWA gives the full diffractive characteristics of the created medium.

\section{ZOGS AS NULLING APS}

In this section, we will focus on the ZOG ability to induce a precise achromatic phase shift (APS stands for Achromatic Phase Shifter) and a controlled intensity balance in order to create a destructive interference as dark as possible. Their implementation into the FQ-PM and AGPM coronagraph will be discussed in the following section. 


\subsection{Preliminary considerations}

The ZOG design procedure consists in the optimization of the $\pi$ phase shift quality within a given spectral range for a well-chosen material. It also requires the equalization of the interfering fluxes, which is difficult to achieve. Indeed, the difference between the effective indices that induces the form birefringence is also responsible for the resultant unequal $T E / T M$-Fresnel reflection coefficients at the interfaces between the modulated region and the external media. The merit function to be minimized is the null depth $N(\lambda)$. The latter is directly related to the phase shift error with respect to $\pi, \epsilon(\lambda)=\Delta \phi_{T E-T M}(\lambda)-\pi$ and the flux ratio $q(\lambda)=\eta_{T E}(\lambda) / \eta_{T M}(\lambda)$ :

$$
N(\lambda)=\frac{(1-\sqrt{q(\lambda)})^{2}+\epsilon(\lambda)^{2} \sqrt{q(\lambda)}}{(1+\sqrt{q(\lambda)})^{2}}
$$

We have investigated several ways to ensure a proper flux balance, i.e. $q \approx 1$. The principle is always the same and consists in trying to minimize the index discontinuities (impedance matching) at the interfaces either by:

1. varying the grating profile: the structure can have trapezoidal profiles instead of rectangular ones. It reduces the parasitic reflection but keeps the anisotropy and a sufficient form birefringence dispersion up to a certain point, unfortunately not enough for our application;

2. "sandwiching" the grating: the superstrate material can be chosen to avoid the brutal air/ZOG interface. ${ }^{9}$ It is conceptually interesting and gives good numerical results but reveals extremely challenging to be manufactured (how to keep the grating openings clear?);

3. depositing a classical anti-reflective (AR) layer: the ZOG is AR treated with a single $\lambda / 4$ layer. The latter settles in the bottom of the openings and on the top of the grating ridges. This leads to an original configuration, a 3-layer grating which complex behaviour is diffraction-governed. We demonstrate that this particular structure can be optimized to give very interesting results.

Presently, subwavelength gratings are manufactured more readily for the infrared domain (near and mid) because there are less and less but still technical difficulties to imprint sub-micron features. Hopefully, the near-term potential applications (VLT-PF and Terrestrial Planet Finder-Coronagraph, the 2015-NASA's space mission) of this technology concern this wavelength domain. We will focus on the astrophysical bandfilters $\mathrm{H}(1.475-$ $1.825 \mu \mathrm{m}), \mathrm{K}(2-2.4 \mu \mathrm{m})$ and $\mathrm{N}(9.3-11.6 \mu \mathrm{m})$. We have now to chose among the restricted list of infrared materials keeping in mind that the ZOG technology is sufficiently flexible to accommodate the majority of them. We will present results for Diamond, Zinc Selenide $(Z n S e)$, Cadmium Telluride $(C d T e)$, Silicon $(S i)$ and Germanium $(G e)$. This choice is justified in three ways: these selected materials are common in infrared applications, they cover a large refractive index spectrum (2-4) and their etching processes are well-known.

\subsection{Theoretical analysis}

The indices for Diamond, ${ }^{17} \mathrm{ZnSe}{ }^{18} \mathrm{CdTe},{ }^{19} \mathrm{Si},{ }^{19} \mathrm{Ge}^{19}$ will be taken from the following representations:

$$
\begin{gathered}
n_{\text {Diamond }, Z n S e, C d T e, G e}(\lambda)=\left(A+\frac{B \lambda^{2}}{\lambda^{2}-C}+\frac{D \lambda^{2}}{\lambda^{2}-E}+\frac{F \lambda^{2}}{\lambda^{2}-G}\right)^{1 / 2} \\
n_{S i}(\lambda)=A+B \lambda+C \lambda^{2}+D \lambda^{3}+E \lambda^{4}
\end{gathered}
$$

The corresponding coefficients are given in Table 1. We assume an ambient temperature $(T=298 K)$, keeping in mind that corrections are needed if $T$ is different. Note that $\lambda$ is expressed in microns in all representations except for Diamond where it is expressed in nanometres. We have performed simulations for the $\mathrm{H}, \mathrm{K}$ and $\mathrm{N}$ bands assuming in each case a ZOG $Y F_{3}$ AR treated. Absorption and dispersion of $Y F_{3}$ have been taken into account (see Fig. 13 in Ref. 20). We have chosen $Y F_{3}$ as a coating material but several other possibilities exist like $B a F_{2}$ and $L a F_{2}$, which could be better choices according to the substrate index. The grating structure in all these simulations consists of a single rectangular profile discretized into three levels to take the deposited 
Table 1. Coefficients for material refractive index representations.

\begin{tabular}{cccc}
\hline Coeff. & Diamond & $\boldsymbol{Z n S e}$ & $\boldsymbol{C d T e}$ \\
\hline A & 1 & 1 & $-2.373 \times 10^{-4} T+3.8466$ \\
B & 0.3306 & 4.46395 & $8.057 \times 10^{-4} T+3.2215$ \\
C & 30625 & 0.0404 & $-1.10 \times 10^{-4} T+0.1866$ \\
D & 4.3356 & 0.46132 & $-2.160 \times 10^{-2} T+12.718$ \\
E & 11236 & 0.1538 & $-3.160 \times 10^{1} T+18753$ \\
F & - & 2.88289 & - \\
G & - & 2213.5 & - \\
\hline Coeff. & \multicolumn{3}{c}{$\boldsymbol{S i}$} \\
A & $1.600 \times 10^{-4} T+3.431$ & $-6.040 \times 10^{-3} T+11.05128$ \\
B & $-2.643 \times 10^{-2}$ & $9.295 \times 10^{-3} T+4.00536$ \\
C & $4.324 \times 10^{-3}$ & $-5.392 \times 10^{-4} T+0.599034$ \\
D & $-3.194 \times 10^{-4}$ & $4.151 \times 10^{-4} T+0.09145$ \\
E & $8.835 \times 10^{-6}$ & $1.51408 T+3426.5$ \\
\hline
\end{tabular}

AR layer into account. The RCWA results are always discussed in terms of the null depth (Eq. 5). Despite the flexibility in the design of the ZOG, there will be a compromise on the substrate refractive properties. Indeed, the dispersion of the form birefringence is proportional to the raw index $n$. Practically, $n$ must be greater than about 2 to sufficiently compensate for the wavelength hyperbolic dependence of the phase shift (Eq. 4) and thus achromatize it. Unfortunately, the intensity of the parasitic reflection phenomenon will also increase accordingly. We already have discussed the flux imbalance problem but the Fresnel reflection will also be responsible for a so-called second order "ghost" in the final coronagraphic image. This ghost comes from the double reflection on the back face of the substrate and on the internal interface of the AR treated ZOG. Its intensity can be quantified assuming a reasonable AR performance for the back side of the component, i.e. about $0.5 \%$ of reflectivity. As the ghost problem increases with the substrate index $n$ (see Fig. 3, top left) and for sake of simplicity, we will restrain our analysis to $Z n S e$ and Diamond, both possessing the lowest index values (near 2.4). Results for both selected materials in terms of null depth are excellent either for the $\mathrm{H}, \mathrm{K}$ or $\mathrm{N}$ bands (Fig. 3). Indeed, the mean null depth performances are at the $10^{-5}$ level over the whole spectral range of the three considered band filters. These optimal results have been calculated by implementing the simplex search method ${ }^{21}$ with our RCWA algorithm. One can see that only minor corrections are needed between the $Z n S e$ and Diamond designs. It is simply due to the fact that their indices are very close though their dispersions are quite different. This level of performances is sufficient in the case of phase coronagraphy as we will see in Sect. 5 .

\section{CORONAGRAPHIC IMPLEMENTATIONS}

\subsection{QZOG}

The ZOG implementation into the FQ-PM is straightforward. Let $\underline{s}$ and $p$ be the vectorial complex amplitude components of the incoming light of wavevector $\underline{k}$. In each of the four quadrants, the $\underline{s}$ and $p$ global polarization states are decomposed in the corresponding $T E_{i}$ and $T M_{i}$ vectorial complex amplitudes according to the local grating line orientations $\left(i=1, \ldots, 4\right.$ is the quadrant number). Two effective indices $n_{T E_{i}}$ and $n_{T M_{i}}$ can be assigned to the corresponding perpendicular polarization states. The four gratings engraved on a unique substrate are strictly identical and implemented in the following way: two of them in two quadrants along one diagonal are rotated by 90 degrees around their normals with respect to the two others. This anti-symmetrical configuration achieves the FQ-PM particular focal plane $\pi$-phase distribution. This new component will be referred as to the Four Quadrant-ZOG, or 4QZOG.

\subsection{AGPM}

The idea of the AGPM coronagraph was to suppress the "shaded zones" resulting for the quadrant transitions of the 4QZOG. The concentric grooves of the AGPM coronagraph are in fact what is called a "space-variant" 

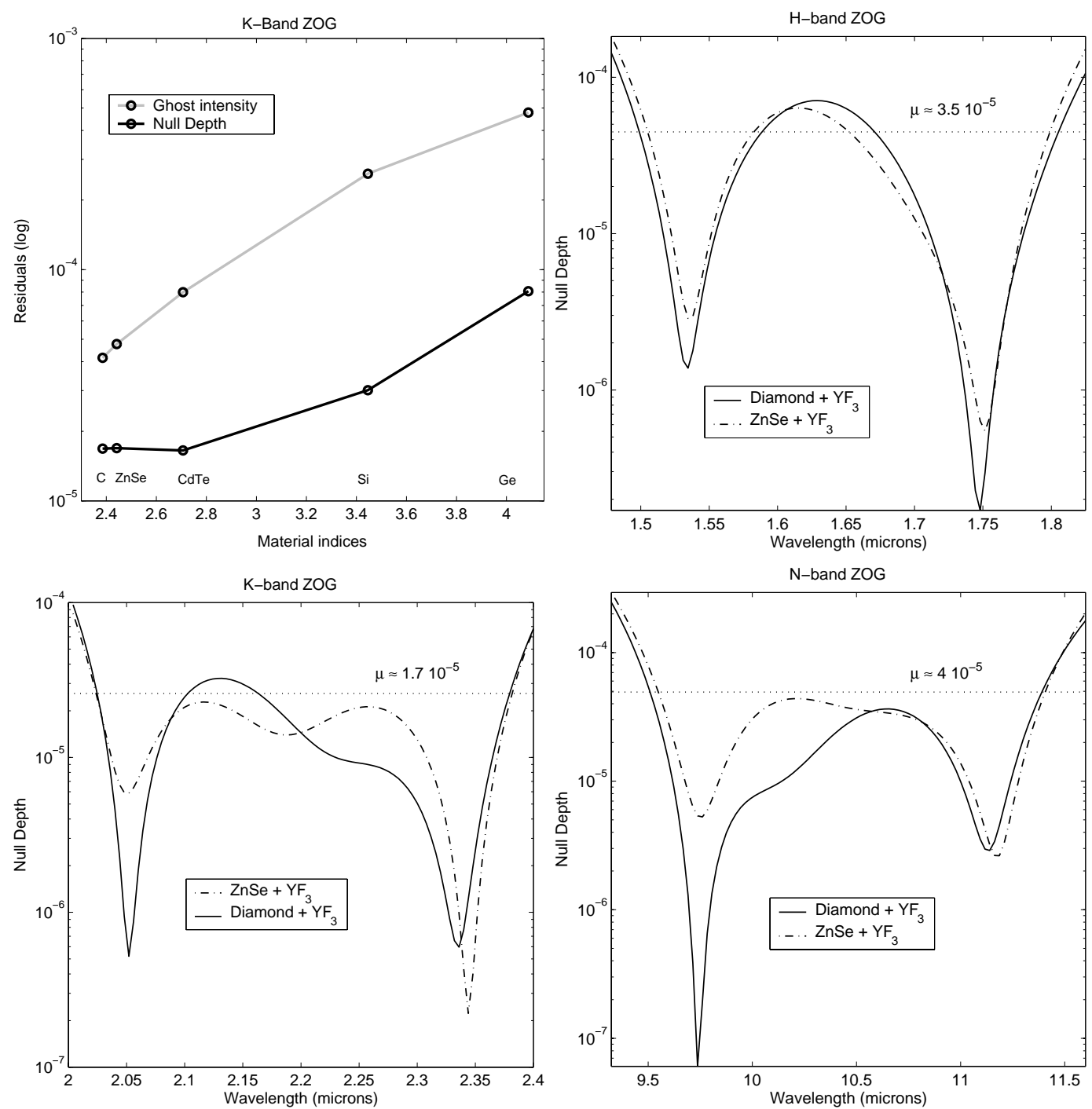

Figure 3. Top left: design level of performance according to the substrate refractive index $n$. The ghost intensity level increases significantly with $n$. We also notice that the rough null depth performance is inversely proportional to $n$. The best compromise is therefore for the low index values. Top right: H-band ZOG Null Depth (logarithmic scale) versus wavelength. The continuous curve is for the Diamond $Y F_{3}$ AR coated 4QZOG. The dashed curve, for the $Z n S e Y F_{3}$ AR coated one. $\mu \approx 3.5 \times 10^{-5}$ is the mean null depth over the whole H band. Bottom left: K-band ZOG Null Depth (logarithmic scale) versus wavelength. The continuous curve is for the Diamond $Y F_{3}$ AR coated 4QZOG. The dashed curve, for the $Z n S e Y F_{3}$ AR coated one. $\mu \approx 1.7 \times 10^{-5}$ is the mean null depth over the whole $\mathrm{K}$ band. Bottom right: N-band ZOG Null Depth (logarithmic scale) versus wavelength. The continuous curve is for the Diamond $Y F_{3}$ AR coated 4QZOG. The dashed curve, for the $Z n S e Y F_{3}$ AR coated one. $\mu \approx 4 \times 10^{-5}$ is the mean null depth over the whole $\mathrm{N}$ band. 


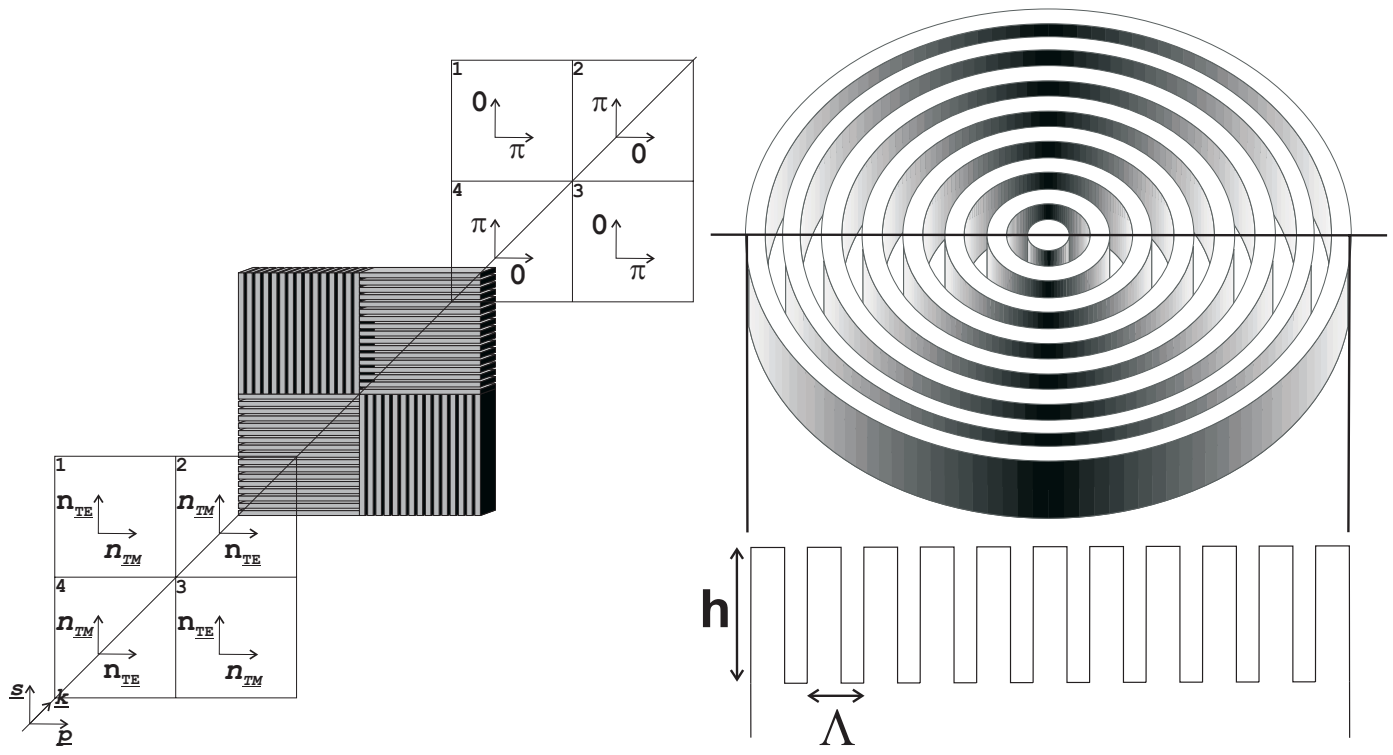

Figure 4. Left: 4QZOG implementation, where the four gratings engraved on a unique substrate are strictly identical and implemented in the following way: two of them in two quadrants along one diagonal are rotated by 90 degrees around their normals with respect to the two others. Right: AGPM implementation. The AGPM consists of a concentric circular surface-relief grating with rectangular grooves with depth $h$ and a periodicity $\Lambda$.

ZOG : when the local characteristics (period, orientation of the grating lines,...) of the structure vary from point to point, it is said to be space-variant. Such components were recently extensively studied as polarization control elements. ${ }^{2-24}$ Applications are numerous: polarimetry, laser-beam shaping, laser machining, tight focusing, particle acceleration, atom trapping, ... In fact, the AGPM space variant ZOG synthesizes a spiral phase plate which creates an "optical vortex". Indeed, at the center of the components, the phase possesses a screw dislocation inducing a phase singularity, i.e. an optical vortex. The central singularity forces the intensity to vanish by a total destructive interference, creating a dark core. This dark core propagates and is conserved along the optical axis. Whether a dark core is created in the pupil or focal plane of a telescope will determine the way it further evolves. In Ref. 8, the author proposes to create an optical vortex in the pupil plane to peer at the faint monochromatic signal in the relayed focal plane with appropriate filtering. In this paper, we propose to do the inverse, i.e. creating an optical vortex in the focal plane, filtering in the relayed pupil plane and making the detection in a final image plane. This solution is theoretically much more attractive. Indeed, unlike the "optical vortex coronagraph" presented in Ref. 8, it can be analytically demonstrated that the theoretical attenuation of the AGPM is infinite in the ideal case (see Ref. 25). Furthermore, the ZOG unique properties permit a broadband use as in the 4QZOG case whereas the component proposed in Ref. 8 is monochromatic. Indeed, the ZOG parameters (period, depth, filling factor) are the same in the 4QZOG and AGPM configurations. As seen before, the only difference concerns the geometry of implementation.

\section{CORONAGRAPHIC SIMULATIONS}

We have performed realistic numerical simulations that imply three different stages:

1. a "Rigorous Coupled Wave Analysis" stage where the form birefringence of the local grating is calculated ;

2. the analytical treatment that gives the spatial distribution of the output linear polarization field (phases and amplitudes) ;

3. a scalar far-field propagation Fourier coronagraphic code for each polarization state. 

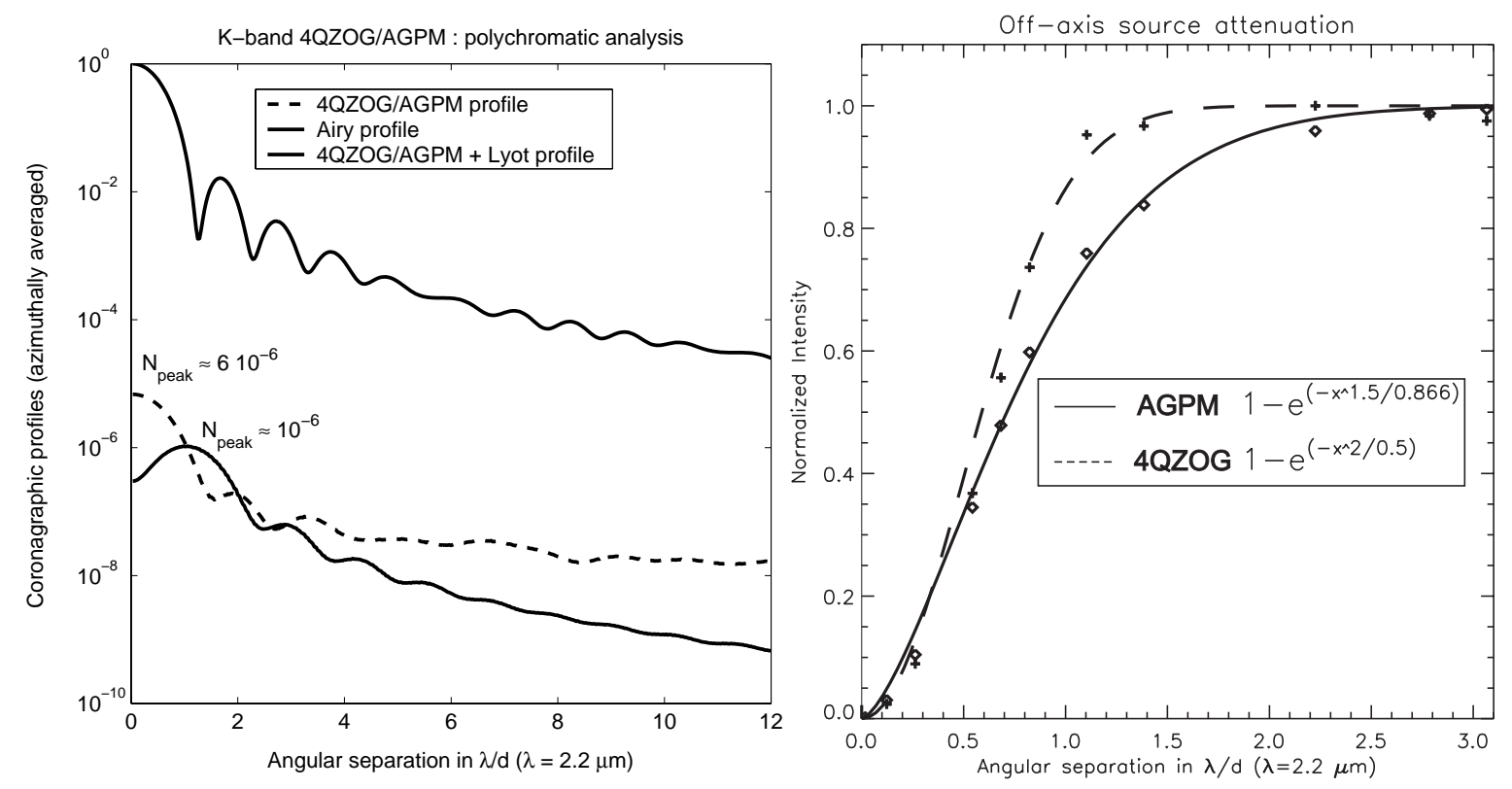

Figure 5. Left: K-band 4QZOG/AGPM coronagraphic profile (logarithmic scale) versus the angular separation in $\lambda_{\text {med }} / D$ unity, $D$ being the telescope diameter. The gray curve corresponds to the polychromatic Airy disk. The dashed one represents the wide band 4QZOG azimuthally averaged profile where the peak-to-peak null depth is about $6 \times 10^{-6}$. The continuous curve corresponds to the previous case with a tiny circular Lyot opaque mask $\left(0.55 \lambda_{\text {med }} / D\right.$ of diameter $)$ to get rid of the chromatic residuals and ghost image. The peak-to-peak null depth is this time about $10^{-6}$. Right: Degradation of the coronagraphic performance vs. angular separation. This figure compares the companion attenuation for the AGPM vs the 4QZOG in its best configuration, i.e. at least $1 \lambda / D$ away from a quadrant transition. This degradation is measured on the total energy. The solid line shows the exponential fit on the simulated data (open losanges) for the AGPM coronagraph. The dashed line shows the exponential fit on the simulated data (crosses) for the 4QPM coronagraph.

For the third stage, we have used an IDL code for Fraunhofer diffraction analysis. ${ }^{12}$ To minimize the aliasing effect of the Fast Fourier Transform, we have used large arrays (up to $2048 \times 2048$ ) for the calculation. The intrinsic nulling performance is only limited by the phase residues around $\pi$ and the differences in transmission between the two polarization states $\underline{T E}$ and $\underline{T M}$. We have also assumed wavefront qualities of $\lambda_{0} / 250, \lambda_{0}$ being the central wavelength of the considered filters. In our case, $\lambda_{0}=2.2 \mu \mathrm{m}$, the wavefront quality assumed is therefore $\approx \lambda_{\text {vis }} / 70$, with $\lambda_{\text {vis }}=632.8 \mathrm{~nm}$. This is quite a severe but somewhat realistic figure. Indeed, the Virgo team ${ }^{26,27}$ has demonstrated state-of-the-art mirror quality with excellent polishing realization $(\lambda / 226 \mathrm{rms}$ @ $632.8 \mathrm{~nm}$ ), within the framework of gravitational wave detection. This technology is directly applicable to our case. The final results of our three-stage calculation are excellent. In both cases, the coronagraphic (for the sake of simplicity, shown here only for the $\mathrm{K}$ band) profile shows a peak-to-peak null depth better than about $\approx 10^{-5}$. The speckle level of $\approx 10^{-7}$ is quickly reached at a few $\lambda / D$ (see Fig. 5 ). The AGPM coronagraphic behavior is very similar to the achromatic 4QZOG coronagraph, but with a total symmetry. Better performances $\left(10^{-6}\right.$ on the peak) can be achieved by placing at the center of the component, a tiny circular Lyot opaque mask of $0.55 \lambda_{\text {med }} / D$ which is the diameter encircling half the Airy disk energy. Doing this, we remove a large part of the ZOG-APS chromatic residuals and also prevent the annoying ghost problem to a certain point. These performances are comfortably one order of magnitude above the H-band/K-Band VLT-PF specification for example. Indeed, the main limitation for ground-based observations comes from the atmospheric turbulence residuals (the so-called speckles) after adaptive optics corrections of the incoming fluctuating wavefronts. In Fig. 5 is presented the attenuation of an off-axis simulated companion in the AGPM vs 4QZOG cases. AGPM behavior is also quite similar to the FQ-PM (thus to the 4QZOG) in its best configuration, i.e. only along the two diagonals. Indeed, as mentioned in the introduction, the FQ-PM/4QZOG quadrant transitions induce a non-negligible attenuation of the superimposed circumstellar features lying on them. These shaded zones 
Table 2. ZnSe or Diamond 4QZOG/AGPM Null Depth level for $\mathbf{H}, \mathbf{K}$ and $\mathbf{N}$ bandfilters: $\left(R_{\lambda}=\lambda / \Delta \lambda\right.$ is the spectral resolution)

\begin{tabular}{|c|c|c|c|}
\hline Filters & $\overline{\mathbf{H}\left(R_{\lambda}=4.7\right)}$ & $\mathbf{K}\left(R_{\lambda}=5.5\right)$ & $\mathbf{N}\left(R_{\lambda}=4.86\right)$ \\
\hline Null Depth (global) & $3.5 \times 10^{-5}$ & $1.7 \times 10^{-5}$ & $4 \times 10^{-5}$ \\
\hline Null Depth $(@ 3 \lambda / D)$ & $2.92 \times 10^{-7}$ & $1.42 \times 10^{-7}$ & $3.3 \times 10^{-7}$ \\
\hline Grating period (Diamond) & $525 \mathrm{~nm}$ & $740 \mathrm{~nm}$ & $3.29 \mu m$ \\
\hline
\end{tabular}

represent quite a significant portion of the focal plane (about $10 \%$ at $6 \lambda / D$ ). Thanks to the perfect AGPM circular symmetry, this problem does not exist any more. Let us emphasize that the Inner Working Angles (IWA) of the 4QZOG and AGPM are very good, peering well under $\lambda / D$. As far as stellar leakage is concerned, numerical simulations show that the AGPM null depth degrades as $\theta_{\lambda / D}^{2}$ close to the optical axis, just as the FQ-PM/4QZOG (with $\theta_{\lambda / D}$, the angle from the optical axis).

\section{MANUFACTURING}

The fabrication of the 4QZOG coronagraph is currently under assessment on Diamond by "Adamantis AB" of the Angström Laboratory ${ }^{28}$ (Uppsala University, Sweden). We have several other contacts with industry for the AGPM. Their manufacturing is based on micro-electronic technologies. The first step consists in imprinting the four quadrant pattern or concentric annular mask in a resin coated on the chosen substrate material. It can be realized by laser direct writing or e-beam lithography. The precision of this step is critical because it defines once and for all the lateral dimensions of the ZOG. For a complete tolerance study, see Ref. 29. This pattern is then uniformly transferred in the substrate by an appropriate reactive plasma beam etching down to the desired depth. The fabrication must be interactive to properly compensate for process errors.

\section{CONCLUSIONS}

In this paper, we have presented two new phase mask coronagraphs that are inherently quasi-achromatic. We have exploited an original solution to the chromaticity problem of the $\pi$ phase shift for the FQ-PM coronagraph by using subwavelength gratings (ZOGs). Indeed, the birefringence of ZOGs can be controlled by the geometry in order to compensate for the hyperbolic dependence of the phase shift and intrinsic material dispersions. Results in terms of global Null Depth are excellent, $\approx 10^{-5}$. The potential performance of the 4QZOG and AGPM coronagraphs is very good ensuring, for instance, a theoretical contrast of $1.4 \times 10^{-7} @ 3 \lambda / D$ over the whole $\mathrm{K}$ band and with an inherent perfect symmetry in the AGPM case. The Inner Working Angle of both masks is $\approx \lambda / D$ with still a good contrast of $\approx 10^{-5}$. Thanks to the ZOG design flexibility, the 4QZOG and AGPM coronagraphs can accommodate a large variety of materials and wavelength bands (see Table 2) thus making them an attractive solution for future ambitious high resolution/high contrast space-(TPF/DARWIN) or ground-(VLT-PF) based imaging in the context of exoplanet detection/characterization. The AGPM configuration should advantageously replace the 4QZOG since the circular symmetry of the vortex prevents any "shaded zone".

\section{ACKNOWLEDGMENTS}

D. Mawet acknowledges the financial support of the Belgian "Fonds pour la formation à la Recherche dans l'Industrie et dans l'Agriculture". P. Riaud and J. Surdej acknowledges the financial support of the "Pôle d'Attraction Inter-Universitaire".

\section{REFERENCES}

1. B.A. Smith and R. Terrile, "A circumstellar disk around Beta Pictoris", Science 226, pp. 1421-1424 (1984).

2. D. Gratadour, D. Rouan, A. Boccaletti, P. Riaud and Y. Clénet, "Four quadrant phase mask K-band coronagraphy of NGC 1068 with NAOS-CONICA at VLT", A $8 A 429$ (2), pp. 433-437 (2005).

3. B. Lyot, "A study of the solar corona and prominences without eclipses", MNRAS 99, pp. 580-594 (1939). 
4. M.J. Kuchner \& D.M. Spergel, "Notch-Filter Masks: Practical Image Masks for Planet-Finding Coronagraphs", ApJ 594, pp. 617-626 (2003).

5. F. Roddier and Cl. Roddier, "Stellar coronagraph with phase mask", PASP 109, pp. 815-820 (1997).

6. D. Rouan, P. Riaud, A. Boccaletti, Y. Clénet and A. Labeyrie, "The four-quadrant phase-mask coronagraph. I. Principle", PASP 112, pp. 1479-1486 (2000).

7. J. Gay \& Y. Rabbia, "Principe d'un coronographe interférentiel", CR. Acad. Sci. Paris 332 (IIb), pp. 265 (1996).

8. G.A. Jr. Swartzlander, "Peering into darkness with a vortex spatial filter", Opt. Lett. 8 (26), pp. 497 (2001).

9. P. Riaud, J. Baudrand, A. Boccaletti and D. Rouan, "The Four-Quadrant Phase-Mask Coronagraph. III. Laboratory Performance", PASP 115, pp. $712-719$ (2003).

10. P. Baudoz, A. Boccaletti, P. Riaud, C. Cavarroc, J. Baudrand, J.M. Rees \& D. Rouan, "The Four-Quadrant Phase-Mask. V. Exoplanet Imaging in the mid-IR with JWST", submitted to PASP (2005).

11. D. Mouillet, T. Fusco, A.M. Lagrange \& J. L. Beuzit, "VLT-"Planet Finder": Specifications for a groundbased high contrast imager", EAS Pub. Ser. 8, pp. 193-196 (2003).

12. P. Riaud, A. Boccaletti, D. Rouan, F. Lemarquis and A. Labeyrie, "The four-quadrant phase-mask coronagraph. II. Simulations", PASP 113, pp. 1145-1154 (2001).

13. H. Kikuta,Y. Ohira and K. Iwata, "Achromatic quarter-wave plates using the dispersion of form birefringence", Applied Optics 36 (7), pp. 1566-1572 (1997).

14. G.P. Nordin and P.C. Deguzman, "Broadband form birefringent quarter-wave plate for the mid-infrared wavelength region", Opt. Express 5 (8), pp. 163-168 (1999).

15. M. Karlsson and Fr. Nikolajeff, "Diamond micro-optics: microlenses and antireflection structured surfaces for the infrared spectral region", Opt. Express 11 (5), pp. 502-507 (2003).

16. M.G. Moharam and T.K Gaylord, "Rigourous Coupled-Wave Analysis of planar grating diffraction", JOSA 71, pp. 811-818 (1981).

17. F. P. Bundy, "Melting Point of Graphite at High Pressure: Heat of Fusion", Science 137, pp. 1055-1057 (1962).

18. W. J. Tropf, "Temperature-dependent refractive index models for BaF2, CaF2, MgF2, SrF2, LiF, NaF, $\mathrm{KCl}$, ZnS and ZnSe", Opt. Eng. 34, pp. 1369-1373 (1995).

19. Gary J. Hawkins, Spectral Characterisation of Infrared Optical Materials and Filters (PhD Thesis - The University of Reading UK, 1998).

20. F. Lemarquis, G. Marchand and C. Amra, "Design and manufacture of low-absorption $Z n S-Y F_{3}$ antireflection coatings in the $3.5-16 \mu \mathrm{m}$ spectral range", Applied Optics 37 (19), pp. 4239-4244 (1998).

21. J.C. Lagarias, J.A. Reeds, M.H. Wright, and P.E. Wright, "Convergence Properties of the Nelder-Mead Simplex Method in Low Dimensions", SIAM Journal of Optimization 9 (1), pp. 112-147 (1998).

22. A. Niv, G. Biener, V. Kleiner, E. Hasman, "Formation of linearly polarized light with axial symmetry by use of space-variant subwavelength gratings", Opt. Lett. 28 (7), pp. 510 (2003).

23. G. Biener, A. Niv, V. Kleiner, E. Hasman, "Formation of helical beams by use of Pancharatnam Berry phase optical elements", Opt. Lett. 27 (21), pp. 1875 (2002).

24. Z. Bomzon, A. Niv, G. Biener, V. Kleiner, E. Hasman, "Polarization Talbot Self-Imaging with ComputerGenerated, Space-Variant Subwavelength Dielectric Gratings", Applied Optics 41 (25), pp. 5218 (2002)

25. D. Mawet, P. Riaud, O. Absil and J. Surdej, "Annular Groove Phase Mask Coronagraph", submitted to ApJ (2005).

26. J.M. Mackowski, L. Pinard, L. Dognin, P. Ganau, B. Lagrange, C. Michel and M. Morgue, "VIRGO mirros: wavefront control", Opt. \& Quant Elect. 31 (5-7), pp. 507-514 (1999).

27. A. Brillet, J.Y. Vinet, V. Loriette, J.M. Mackowski, L. Pinard, A. Remillieux, "Virtual gravitational wave interferometers with actual mirrors", Phys. Rev. D 67, 102006

28. M. Karlsson, K. Hjort, Fr. Nikolajeff, "Transfer of continuous-relief diffractive structures into diamond by use of inductively coupled plasma dry etching", Opt. Lett. 26 (22), 1752-1754 (2001).

29. D. Mawet, P. Riaud, J. Baudrand and J. Surdej, "Subwavelength surface-relief gratings for stellar coronagraphy", to appear in Applied Optics (accepted) (2005). 